Review Article

\title{
Mechanism of Follicular Helper T Cell Differentiation Regulated by Transcription Factors
}

\author{
Long-Shan Ji, ${ }^{1}$ Xue-Hua Sun $\mathbb{D}^{2},{ }^{2}$ Xin Zhang, ${ }^{1}$ Zhen-Hua Zhou, ${ }^{1}$ Zhuo Yu, ${ }^{2}$ Xiao-Jun Zhu $\left(\mathbb{D},{ }^{2}\right.$ \\ Ling-Ying Huang, ${ }^{2}$ Miao Fang, ${ }^{1}$ Ya-Ting Gao, ${ }^{1}$ Man Li $\left(\mathbb{1},{ }^{1}\right.$ and Yue-Qiu Gao ${ }^{1}{ }^{1}$ \\ ${ }^{1}$ Laboratory of Cellular Immunity, Institute of Clinical Immunology, Shanghai Key Laboratory of Traditional Chinese \\ Clinical Medicine, Shuguang Hospital, Affiliated to Shanghai University of Traditional Chinese Medicine, Shanghai 201203, China \\ ${ }^{2}$ Department of Hepatopathy, Shuguang Hospital, Affiliated to Shanghai University of Traditional Chinese Medicine, \\ Shanghai 201203, China
}

Correspondence should be addressed to Man Li; liman121000@126.com and Yue-Qiu Gao; gaoyueqiu@hotmail.com

Long-Shan Ji and Xue-Hua Sun contributed equally to this work.

Received 22 April 2020; Accepted 15 June 2020; Published 20 July 2020

Guest Editor: Margarete D. Bagatini

Copyright (C) 2020 Long-Shan Ji et al. This is an open access article distributed under the Creative Commons Attribution License, which permits unrestricted use, distribution, and reproduction in any medium, provided the original work is properly cited.

Helping B cells and antibody responses is a major function of $\mathrm{CD} 4^{+} \mathrm{T}$ helper cells. Follicular helper $\mathrm{T}$ (Tfh) cells are identified as a subset of $\mathrm{CD} 4^{+} \mathrm{T}$ helper cells, which is specialized in helping B cells in the germinal center reaction. Tfh cells express high levels of CXCR5, PD-1, IL-21, and other characteristic markers. Accumulating evidence has demonstrated that the dysregulation of Tfh cells is involved in infectious, inflammatory, and autoimmune diseases, including lymphocytic choriomeningitis virus (LCMV) infection, inflammatory bowel disease (IBD), systemic lupus erythematosus (SLE), rheumatoid arthritis (RA), IgG4-related disease (IgG4-RD), Sjögren syndrome (SS), and type 1 diabetes (T1D). Activation of subset-specific transcription factors is the essential step for Tfh cell differentiation. The differentiation of Tfh cells is regulated by a complicated network of transcription factors, including positive factors (Bcl6, ATF-3, Batf, IRF4, c-Maf, and so on) and negative factors (Blimp-1, STAT5, IRF8, Bach2, and so on). The current knowledge underlying the molecular mechanisms of Tfh cell differentiation at the transcriptional level is summarized in this paper, which will provide many perspectives to explore the pathogenesis and treatment of the relevant immune diseases.

\section{Introduction}

$\mathrm{CD} 4^{+}$helper $\mathrm{T}$ cells play a critical role in forming and amplifying the abilities of the immune system. Follicular helper $\mathrm{T}$ (Tfh) cells are identified as a subset of $\mathrm{CD} 4^{+} \mathrm{T}$ helper cells, which provides help to $\mathrm{B}$ cells for the formation and maintenance of the germinal center (GC), the production of high affinity class-switched antibodies, long-lived plasma cells, and memory B cells [1]. There were a great deal of researches about Tfh cells in the past 10 years; in particular, the differentiation and function of Tfh cells were involved in a range of diseases including infectious diseases, vaccines, autoimmune diseases, and allergies. Tfh cells are characterized by high expression of the chemokine receptor CXCR5, the transcription factor Bcl6, the costimulatory molecule ICOS, and the coinhibitory molecule $\mathrm{PD}-1$. Once naïve $\mathrm{CD} 4^{+} \mathrm{T}$ cells are activated by antigen-presenting cells (APCs) together with IL-6 and IL-21, they will differentiate into Tfh cells.

A multiple-stage process is involved in the generation of Tfh cells from naïve $\mathrm{CD} 4^{+} \mathrm{T}$ cells, which consists of initiation, maintenance, and full polarization stages [1]. During the initiation phase of Tfh cell differentiation, multiple signals take part in the process, including transcription factors (Bcl6, Ascl2, Batf, IRF4, c-Maf, and so on), costimulatory molecule(ICOS), and cytokines(IL-6/IL-21); in particular, higher TCR affinity is necessary for initiation of Tfh cell 
$\left(\mathrm{Bcl}^{+} \mathrm{CXCR} 5^{+}\right)$differentiation at the phase of dendritic cell priming [2-7]. Then, $\mathrm{Bcl}^{+} \mathrm{CXCR}^{+}$Tfh precursor cells move into the T-B border zone, where they accept other differentiation signals from activated B cells [8]. After this appointment, the toughened expression of Bcl6 regulates surface markers, which accelerates the migration of Tfh cells into GC, where they offer assistant signals for B cells $[9,10]$ (Figure 1).

Differentiation of naïve $\mathrm{CD}^{+} \mathrm{T}$ cells into $\mathrm{Tfh}$ cells is modulated by a multipart transcriptional network (Figure 2). Multiple transcription factors that either support or oppose the differentiation and function of Tfh cells have been identified (Table 1).

Now, the knowledge of the transcriptional mechanism underlying Tfh cell differentiation will be comprehensively described in this paper, which will highlight the possible future directions.

\section{Bcl6 and Blimp-1}

Bcl6 has been known as a key transcription factor for Tfh cell development by pathways essentially independent of Blimp$1[3,39]$. Bcl6 consists of a zinc finger domain; a bric-a-brac, tramtrack, broad-complex (BTB) domain; and a middle domain [40]. The Bcl6 DNA binding zinc finger domain is essential for Bcl6 activity in $\mathrm{CD}^{+} \mathrm{T}$ cells [8]. The BTB domain of $\mathrm{Bcl} 6$ participates in the correct differentiation of Tfh cells most likely by interacting with Bcl6-interacting corepressor (BCOR) [41]. The middle domain of Bcl6 prevents the association with the corepressor metastasisassociated protein 3 (MTA3) and inhibits the differentiation and function of Tfh cells by distressing Prdm1 (encodes Blimp-1) and other crucial target genes [42].

Bcl6 expression is induced by IL-6-STAT1/STAT3 signaling [43], and it is driven very early after T cell activation in a CD28-dependent manner [44]. The E3 ubiquitin ligase Itch is essential for Bcl6 expression at the early stage of Tfh cell development [45]. The deficiency of the WiskottAldrich syndrome protein suppresses Bcl6 transcription, which results in a deficient response of Tfh cells [46]. Research shows that Bcl6 inhibits the IL-7R/STAT5 axis during Tfh cell generation [47]. Bcl6 mediates the effect of activating transcription factor 3 (ATF-3) on Tfh cells in the gut [16]. ATF-3 is a stress-inducible transcription factor and plays a critical role in the prevention of colitis by regulating the development of Tfh cells in the gut. In addition, Bcl6 also suppresses the expression of specific microRNAs that are thought to control the differentiation of Tfh cells, such as miR-17-92 [9] and miR-31 [48]. The miR-17-92 inhibits CXCR5 expression, and miR-31 directly binds to Bcl6 promoter.

Blimp-1 has been found to be a critical transcriptional repressor for Tfh cell differentiation. Blimp-1 has the inhibitory effect on $\mathrm{Bcl} 6$ expression, indicating that $\mathrm{Bcl} 6$ and Blimp-1 are antagonistic regulators in the differentiation of Tfh cells. Blimp-1 is induced by IL-2/STAT5 signaling, and it suppresses the expression of Tfh-associated genes including Bcl6, c-Maf, Batf, CXCR5, and IL-21 [25, 26]. Blimp-1deficient $\mathrm{CD}^{+} \mathrm{T}$ cells in mice show enhanced $\mathrm{Tfh}$ cell differentiation and GC formation [3, 49]. Taken together, these results indicate that Bcl6 is both necessary and sufficient for Tfh cell development and the proper differentiation of Tfh cells in vivo, and the differentiation of Tfh cells requires keeping the expression balance between Bcl6 and Blimp-1.

Bcl6 and Blimp-1 are associated with various infectious and autoimmune diseases by regulating Tfh cells. Bcl6 is highly expressed in sinus tissues, parotid gland tissues, and lacrimal gland tissues of IgG4-related disease (IgG4-RD) patients [14]. Blimp-1 in peripheral blood is upregulated in patients with IgG4-RD [14]. Compared with the healthy controls, higher expression of Bcl6 and lower Blimp-1 expression in the peripheral blood are observed in patients with rheumatoid arthritis (RA) [15].

\section{3. c-Maf and Batf}

c-Maf and Batf are the members of the activator protein 1 (AP-1) family. c-Maf is a bZIP transcriptional factor, and promotes the differentiation of Tfh cells. [6]. It is highly expressed in Th17 cells and mature Tfh cells. The selective loss of c-Maf expression in Tfh cells results in the downregulated expression of Bcl6, CXCR5, PD-1, and IL-21 [6]. In addition, one study reveals that Bcl6 and c-Maf synergistically orchestrate the expression of Tfh cell-associated genes (PD-1, ICOS, CXCR5, and so on) [4].

Batf is known to control switched antibody responses. Batf is highly expressed in Tfh cells and is essential for the differentiation of Tfh cells through regulating the expression of Bcl6 and c-Maf $[50,51]$. Batf directly binds to and activates the conserved noncoding sequence 2 (CNS2) region in the IL-4 locus and then triggers the production of IL- 4 in Tfh cells [52].

Both c-Maf and Batf are related with immune diseases. Compared with the healthy controls, c-Maf mRNA expression level and percentage of Tfh cells in peripheral blood mononuclear cells (PBMCs) are increased in patients with chronic immune thrombocytopenia (cITP), and they are decreased after the effective treatment [12]. Compared with the healthy controls, Batf in the submandibular glands and affected lymph nodes is markedly increased in patients with IgG4-RD [17].

\section{IRF4 and IRF8}

IRF4 and IRF8 belong to the evolutionarily conserved IRF family. IRF4 is expressed in hematopoietic cells and plays pivotal roles in the immune response. It has been acknowledged that the IRF4 locus "senses" the intensity of TCR signaling to determine the expression level of IRF4 [18]. IRF4 plays a critical role in regulating the generation of Tfh cells. In $\mathrm{IRF}^{-/-}$mice, $\mathrm{CD}^{+} \mathrm{T}$ cells in lymph nodes and Peyer's patches fail to express Bcl6 and other Tfh-related molecules [53]. IL-21 is a key cytokine for the development of Tfh cells [54], and IRF4 regulates the production of IL-21 [55]. Therefore, IL-21 takes part in regulating the differentiation of Tfh cells by IRF4. In wild-type mice, IRF4 can interact with Batf-JUN family protein complexes to form a heterotrimer 


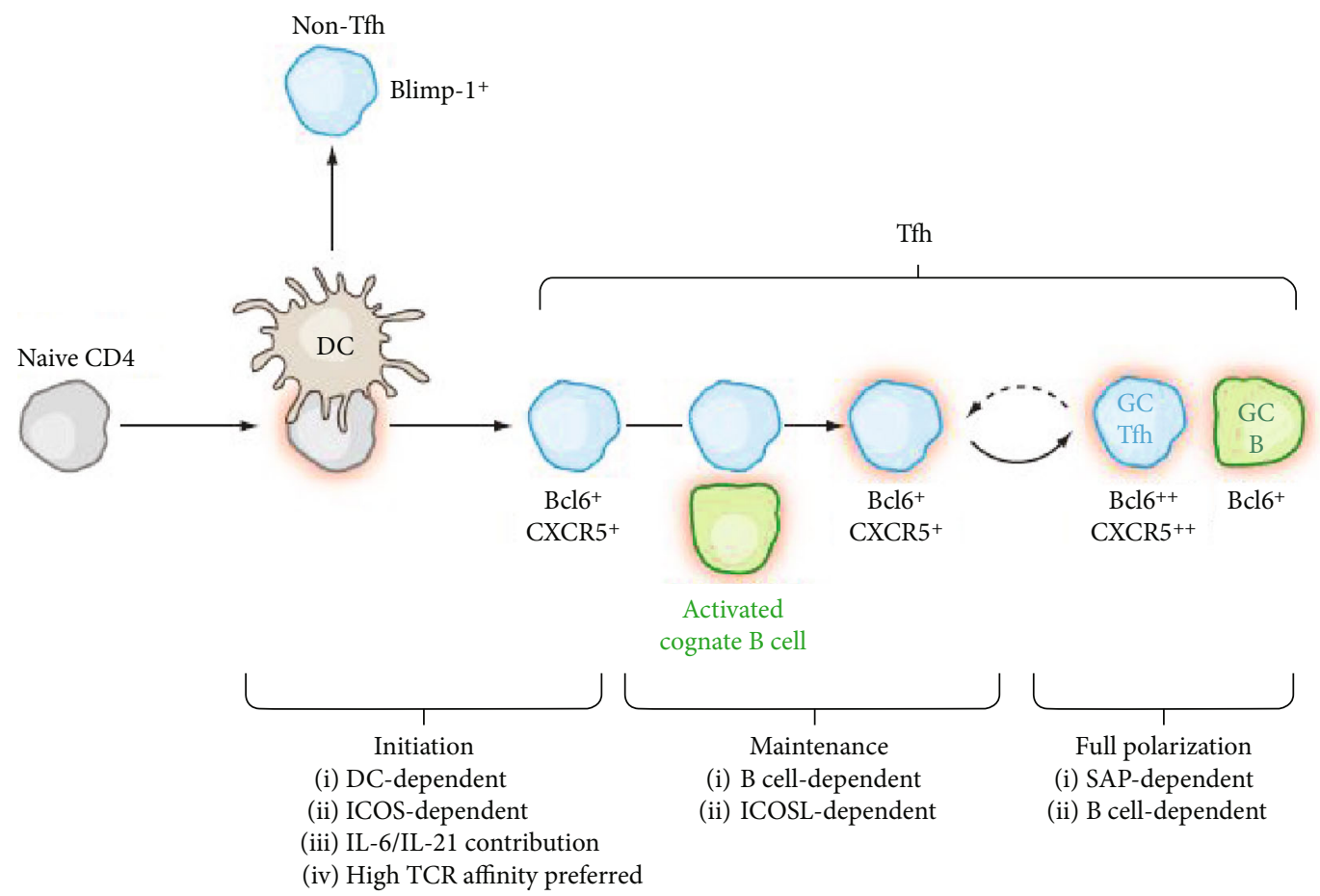

FIGURE 1: The differentiation of Tfh cells: multiple stages of Tfh cell differentiation, including initiation, maintenance, and full polarization stages (Annual Review of Immunology, 2011,29(1):621-663).

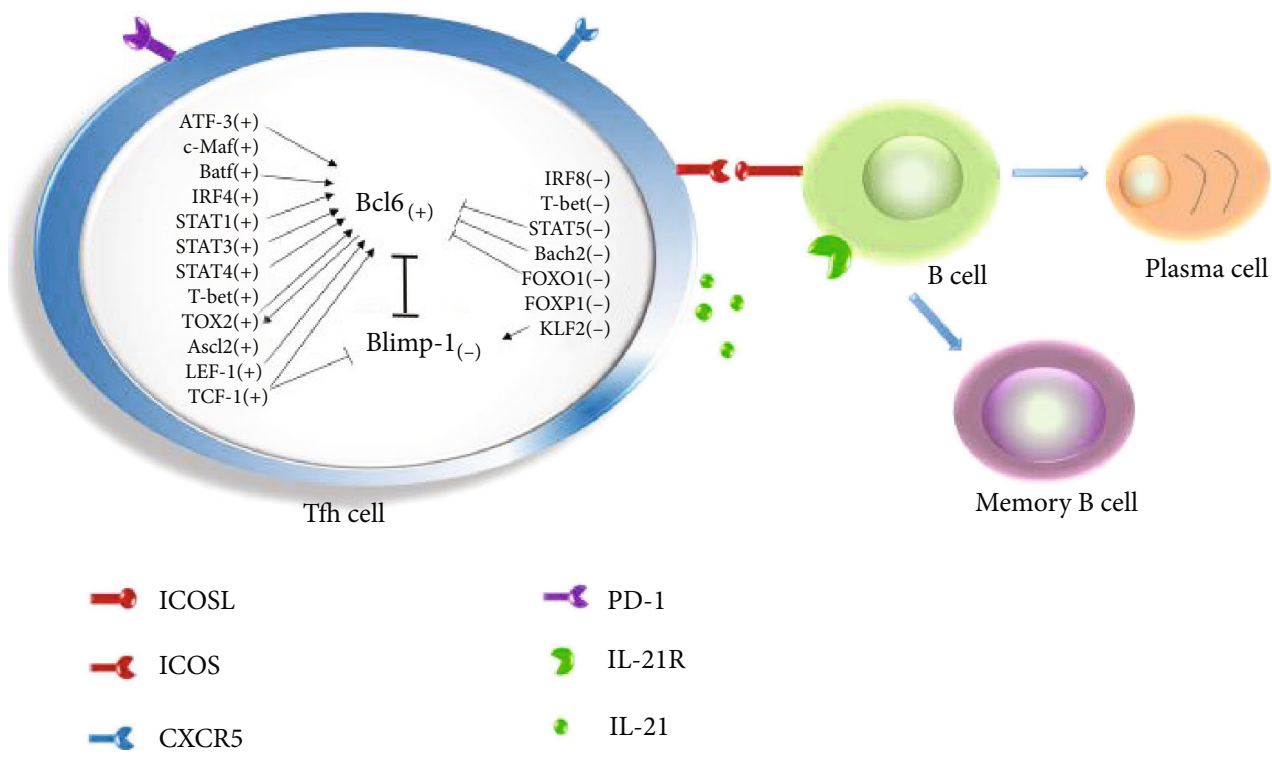

Figure 2: Network of transcription factors in the differentiation of Tfh cells. Tfh cells are regulated by a complex network of transcription factors, including Bcl6, Blimp-1, ATF-3, c-Maf, Batf, IRF4, IRF8, STATs, T-bet, TOX2, Ascl2, LEF-1, TCF-1, Bach2, FOXO1, FOXP1, and KLF2. "+" means positive factors and "-" means negative factors. " $\rightarrow$ " means the promoting effect and " $\dashv$ ” means the inhibitory effect.

that can bind to AP1-IRF4 complexes and regulate Th cell differentiation $[50,51]$.

IRF8 plays various and important regulatory roles in the growth, differentiation, and function of immune cells in inflammatory bowel disease (IBD) patients [19]. IRF8 inhibits the differentiation of Tfh cells by directly binding to the promoter region of the IRF4 gene and inhibiting the transcription and activation of IRF4. In contrast, IRF8 defi- ciency significantly enhances IRF4 binding the promoter region of the IL-21 gene and results in the expansion of Tfh cell differentiation in vitro and in vivo [19].

\section{STATs}

Members of the STAT family including STAT1, STAT3, STAT4, and STAT5 are the important regulators for the 
TABle 1: Transcription factors in the differentiation of Tfh cells.

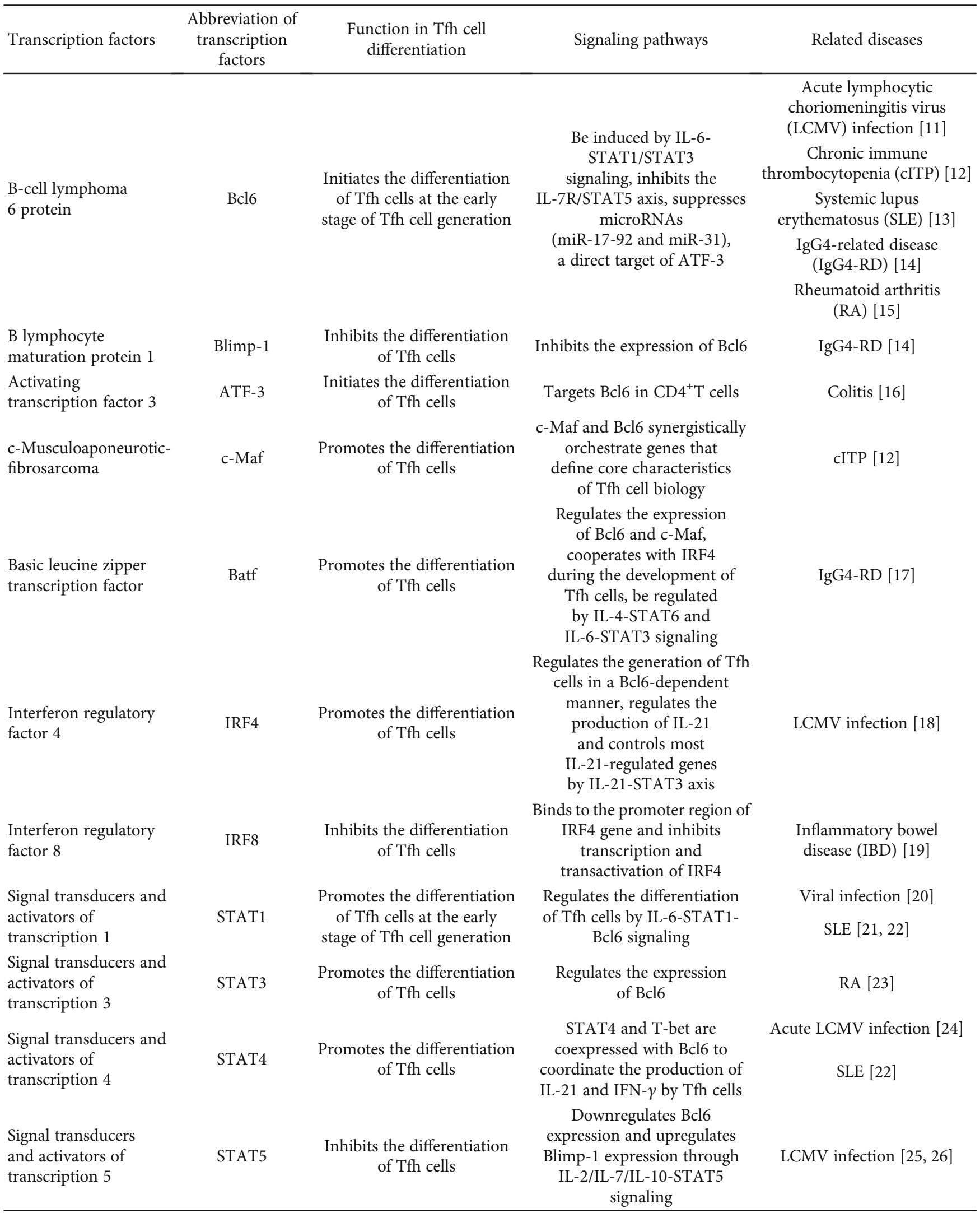


TABLE 1: Continued.

\begin{tabular}{|c|c|c|c|c|}
\hline Transcription factors & $\begin{array}{l}\text { Abbreviation of } \\
\text { transcription } \\
\text { factors }\end{array}$ & $\begin{array}{l}\text { Function in Tfh cell } \\
\text { differentiation }\end{array}$ & Signaling pathways & Related diseases \\
\hline $\begin{array}{l}\text { T-box expressed in } \\
\mathrm{T} \text { cells }\end{array}$ & T-bet & $\begin{array}{l}\text { Mildly inhibits the early } \\
\text { differentiation of Tfh cells, } \\
\text { but promotes Tfh cell } \\
\text { proliferation and apoptotic } \\
\text { intervention at } \\
\text { the late effector phase }\end{array}$ & $\begin{array}{l}\text { T-bet and STAT4 } \\
\text { are coexpressed } \\
\text { with Bcl6 to coordinate the } \\
\text { production of IL- } 21 \text { and } \\
\text { IFN- } \gamma \text { by Tfh cells }\end{array}$ & LCMV infection $[2,24,27]$ \\
\hline $\begin{array}{l}\text { T cell-specific } \\
\text { transcription factor } 1\end{array}$ & TCF-1 & $\begin{array}{l}\text { Promotes the differentiation } \\
\text { of Tfh cells at the early stage } \\
\text { of Tfh cell generation }\end{array}$ & $\begin{array}{l}\text { Promotes the expression of } \\
\text { Bcl6, but represses the } \\
\text { expression of Blimp-1 }\end{array}$ & LCMV infection $[7,28-30]$ \\
\hline $\begin{array}{l}\text { Lymphoid enhancer } \\
\text { binding factor } 1\end{array}$ & LEF-1 & $\begin{array}{l}\text { Promotes the differentiation } \\
\text { of Tfh cells at the early } \\
\text { stage of Tfh cell generation }\end{array}$ & $\begin{array}{l}\text { Works synergistically with } \\
\text { TCF-1 to enhance the } \\
\text { expression of ICOS and Bcl6 }\end{array}$ & LCMV infection [30] \\
\hline $\begin{array}{l}\text { The high-mobility } \\
\text { group- (HMG-) box } 2\end{array}$ & TOX2 & $\begin{array}{c}\text { Initiates the differentiation } \\
\text { of Tfh cells }\end{array}$ & $\begin{array}{l}\text { Be regulated by Bcl6 and } \\
\text { STAT3 in the initial stage } \\
\text { of Tfh cell generation, } \\
\text { inhibits IL-2 and/or } \\
\text { enhances IL- } 6 \text { signaling } \\
\text { to promote Bcl6 expression }\end{array}$ & Viral infection [31] \\
\hline $\begin{array}{l}\text { Achaete-scute } \\
\text { homolog } 2\end{array}$ & Ascl2 & $\begin{array}{c}\text { Promotes the differentiation } \\
\text { of Tfh cells }\end{array}$ & $\begin{array}{l}\text { Upregulates CXCR5 but } \\
\text { not Bcl6 and downregulates } \\
\text { CCR7 expression as well } \\
\text { as IL-2 signaling }\end{array}$ & Sjögren syndrome (SS) [32] \\
\hline $\begin{array}{l}\text { BTB and CNC } \\
\text { homolog } 2\end{array}$ & Bach2 & $\begin{array}{c}\text { Inhibits the differentiation } \\
\text { of Tfh cells }\end{array}$ & $\begin{array}{l}\text { Suppresses the expression of } \\
\text { Bcl6 by directly binding to } \\
\text { the promoter, negatively } \\
\text { regulates CXCR } 5 \text { expression }\end{array}$ & Viral infection $[33,34]$ \\
\hline $\begin{array}{l}\text { Forkhead-box } \\
\text { protein } \mathrm{O} 1\end{array}$ & FOXO1 & $\begin{array}{l}\text { Inhibits the differentiation } \\
\text { of Tfh cells }\end{array}$ & $\begin{array}{l}\text { Negatively regulates the } \\
\text { differentiation of Tfh cells } \\
\text { through an ICOS-mTORC2- } \\
\text { FOXO1 signaling axis } \\
\text { in the early stages of } \\
\text { differentiation, } \\
\text { negatively regulates the } \\
\text { expression of Bcl6 }\end{array}$ & $\begin{array}{l}\text { Angioimmunoblastic } \\
\text { T cell lymphoma } \\
\text { induced [35] }\end{array}$ \\
\hline $\begin{array}{l}\text { Forkhead-box } \\
\text { protein P1 }\end{array}$ & FOXP1 & $\begin{array}{c}\text { Inhibits the differentiation } \\
\text { of Tfh cells }\end{array}$ & $\begin{array}{l}\text { Negatively regulates the } \\
\text { expression of CTLA- } 4 \\
\text { and IL- } 21 \text { in activated } \\
\text { CD } 4^{+} \mathrm{T} \text { cells }\end{array}$ & LCMV infection [36] \\
\hline Krüppel-like factor 2 & KLF2 & $\begin{array}{l}\text { Inhibits the differentiation } \\
\text { of Tfh cells }\end{array}$ & $\begin{array}{l}\text { Downregulates S1PR1, induces } \\
\text { the expression of Blimp-1, } \\
\text { miRNA92a-mediated } \\
\text { Tfh precursor induction } \\
\text { is regulated by } \\
\text { PTEN-PI3K-KLF2 signaling }\end{array}$ & $\begin{array}{l}\text { Type } 1 \text { diabetes } \\
\text { (T1D) [37] }\end{array}$ \\
\hline
\end{tabular}

generation of Tfh cells $[43,54]$. STAT1 is necessary for IL-6mediated Bcl6 induction during the early differentiation of Tfh cells [43]. STAT3 has been found to be critical for Tfh cell development in a Bcl6-dependent manner [23]. The major STAT3-stimulating cytokines include IL-6, IL-21, IL12 , IL-10, and TGF- $\beta[23,56,57]$. Besides, STAT3 regulates Bcl6 expression by cooperating with the Ikaros zinc finger transcription factors Aiolos and Ikaros [58]. TRAF6 inhibits the activation of type I interferon-STAT3 signaling [59]. The latest research clearly shows that T-bet, although mildly inhi- biting early Tfh cell differentiation, mainly plays a crucial and specific supporting role for Tfh cell response by promoting cell proliferation and apoptotic intervention at the endstage effector phase of acute viral challenge [2]. T-bet and STAT4 are coexpressed with Bcl6 to coordinate the production of IL-21 and IFN- $\gamma$ by Tfh cells and promote the GC response [24]. STAT5 is shown as an inhibitory factor for the differentiation of Tfh cells. Molecular analyses reveal that the activation of the IL-2/STAT5 signaling enhances the expression of Blimp-1 and prevents the binding of STAT3 
to the Bcl6 locus [25], resulting in the decrease of GC and the long-lived antibody responses [26]. Similarly, IL-7dependent activation of STAT5 contributes to Bcl6 repression [60]. The latest research shows that IL-10 suppresses the differentiation of Tfh cells in human and mice by promoting STAT5 phosphorylation [61]. He et al. [62] demonstrate that the secreted protein extracellular matrix protein 1 induced by IL- 6 and IL- 21 in Tfh cells promotes the differentiation of Tfh cells by downregulating the level of STAT5 phosphorylation and upregulating Bcl6 expression.

T-bet and STATs are the important regulators for Tfh cell development in infectious and autoimmune diseases. STAT1 serine-727 phosphorylation (designated STAT1-pS727) plays an important role in promoting Tfh cell responses, leading to systemic lupus erythematosus- (SLE-) associated autoantibody production [21]. Compared with the healthy controls, the expression levels of pSTAT1, pSTAT4, and Tbet in PBMCs are upregulated in patients with SLE [22]. The expression level of pSTAT3 in PBMCs in patients with $\mathrm{RA}$ is higher than that in healthy controls [23].

\section{TCF-1 and LEF-1}

TCF-1 is expressed in both developing and mature T cells and is essential for initiating and securing the differentiation of Tfh cells $[7,63]$. TCF-1 directly binds to the Bcl6 transcription start site and Prdm1 $5^{\prime}$ regulatory regions, which promotes the expression of Bcl6 and represses the expression of Blimp-1 during acute viral infection [7, 28, 29]. TCF-1 synergistically works with LEF- 1 to promote the early differentiation of $\mathrm{Tfh}$ cells by the multipronged approach of maintaining the expression of IL-6Ra and gp130, enhancing the expression of ICOS, and promoting the expression of Bcl6 [30].

\section{TOX2}

The high-mobility group- (HMG-) box transcription factor TOX2 is selectively expressed in human Tfh cells and regulated by Bcl6 and STAT3 in the initial stage of Tfh cell generation [31]. There is a feed-forward loop centering on TOX2 and Bcl6, which drives Tfh cell development. TOX2 promotes Bcl6 expression by inhibiting IL-2 and/or enhancing IL-6 signaling during Tfh cell development. Furthermore, TOX2 is bound to the sites shared by Batf and IRF4, which suggests that TOX2, Batf, and IRF4 may functionally converge in developing Th cells.

\section{Ascl2}

Ascl2, a basic helix-loop-helix domain-containing transcription factor, is highly expressed in Tfh cells, and its expression may precede Bcl6 expression. The expression of Ascl2 in the spleen is upregulated in sjögren syndrome (SS) model mice compared with control mice [32]. Ascl2 initiates the differentiation of Tfh cells via upregulating CXCR5 and downregulating $\mathrm{C}-\mathrm{C}$ chemokine receptor 7(CCR7) expression as well as the IL-2 level in T cells in vitro. The Ikappa BNS is highly expressed in Tfh cells and is essential for Ascl2-induced
CXCR5 expression during the differentiation of Tfh cells [64]. After activation of the signals related to Tfh cells described above, Ascl2 accelerates $\mathrm{T}$ cell migration into the follicles in mice [5]. Acute deletion of Ascl2, as well as inhibition of its function with the Id3 protein, can result in impaired Tfh cell development and GC response [5]. In addition, epigenetic regulations, such as histone modifications, also coordinately control the differentiation and function of Tfh cells along with transcription factors. The Ascl2 locus is marked with the active chromatin marker trimethylated histone $\mathrm{H} 3$ lysine 4 (H3K4me3) in Tfh cells, and other transcription factors including Bcl6, Maf, Batf, and IRF4 are uniformly associated with H3K4me3 [65].

\section{Bach2}

Bach2 is a negative regulator of Tfh cell differentiation. Bach2 directly represses the expression of Bcl6 by inhibiting Bcl6 promoter activity [34] and negatively regulates CXCR5 expression [34]. Overexpression of Bach2 in Tfh cells inhibits the expression of Bcl6, IL-21, and the coinhibitory receptor TIGIT [34]. The deletion of Bach2 leads to the upregulation of CXCR5 expression and contributes to preferential Tfh cell differentiation [33].

\section{FOXO1 and FOXP1}

FOXO1 has been found to negatively regulate the differentiation of Tfh cells through the ICOS-mTORC2-FOXO1 signaling in the early stage of differentiation [66]. FOXO1 regulates the differentiation of Tfh cells by negatively regulating Bcl6. The E3 ubiquitin ligase Itch is essential for the differentiation of Tfh cells. Itch associates with FOXO1 and promotes its ubiquitination and degradation [45] and then positively regulates the differentiation of Tfh cells. FOXP1 negatively regulates the expression of CTLA- 4 and IL-21 in activated $\mathrm{CD} 4^{+} \mathrm{T}$ cells [36]. Naïve $\mathrm{CD} 4^{+} \mathrm{T}$ cells deficient in the FOXP1 preferentially differentiate into Tfh cells, which results in substantially enhanced GC and antibody responses [67]. In addition, FOXP1-deficient Tfh cells restore the generation of high-affinity Abs when cocultured with high numbers of single clone B cells [36].

\section{KLF2}

The transcription factor KLF2 serves to inhibit Tfh cell generation by downregulating sphingosine-1-phosphate receptor (S1PR1). KLF2 deficiency in activated $\mathrm{CD}^{+}{ }^{+} \mathrm{T}$ cells contributes to Tfh cell generation, whereas KLF2 overexpression prevents Tfh cell production. KLF2 also induces the expression of Blimp-1 and thereby inhibits the differentiation of Tfh cells [38]. ICOS maintains the phenotype of Tfh cells by downregulating KLF2. KLF2 is identified as a target of miRNA92a in inducting the expression of the human Tfh precursor, and the miRNA92a-mediated Tfh precursor induction is regulated by PTEN-PI3K-KLF2 signaling [37]. 


\section{Conclusions}

Multiple transcription factors have been found to regulate Tfh cell generation. In this paper, the regulatory mechanisms of transcription factors on Tfh cell differentiation are summarized. However, many questions remain to be further investigated. (i) Are there other Tfh-specific transcription factors beyond the abovementioned factors? (ii) How do Tfh-specific transcriptional factors impact epigenetic mechanisms during inducing Tfh cell generation? (iii) What are the factors' stage-specific requirements? (iv) What are the molecular mechanisms contributing to Tfh cell maintenance and memory formation?

As summarized in this review, Tfh cell-related transcription factors including Bcl6, IRF4, STAT1/STAT4/STAT5, Tbet, TCF-1, LEF-1, TOX2, Bach2, FOXP1, and KLF2 are all involved in the virus infection. Both Bcl6 and STAT3 play an important role in RA. ="The expression levels of Bcl6, STAT1, STAT4 and T-bet are upregulated in SLE patients. Bcl6, Blimp-1, and Batf are associated with IgG4-RD. Due to the association of Tfh cells with a broad spectrum of diseases, subsequent in-depth investigation of regulatory factors for the differentiation of Tfh cells may provide the potential therapeutic targets for various immune diseases, especially the virus infection, SLE, RA, and IgG4-RD.

\section{Conflicts of Interest}

The authors declare that they have no conflicts of interest.

\section{Authors' Contributions}

Long-shan Ji and Xue-hua Sun contributed equally to this work.

\section{Acknowledgments}

This work was supported by the National Natural Science Foundation of China (81673767, 81673938, 81673935, 81774240, 81874436, and 81973773); Training Plan for Excellent Academic Leaders of Shanghai Health System (2017BR007); Municipal Hospital Emerging Cutting Edge Technology Joint Research Project (SHDC12016121); Science Research Project of Thirteen Five-Year Plan (2018ZX10725-504); "Shuguang Program" supported by the Shanghai Education Development Foundation and Shanghai Municipal Education Commission (18SG39); Shanghai Sailing Program (20YF1450200); Training Plan of Outstanding Young Medical Talents, Shanghai Municipal Health Bureau (2018YQ43); Three-Year Action Plan of Development of TCM in Shanghai (ZY(2018-2020)-FWTX-4001, ZY(2018-2020)-FWTW-4004); Pilot Project of Clinical Cooperation between Chinese and Western Medicine for Major and Difficult Diseases (Hepatic Fibrosis); and Si Ming Foundation of Shuguang Hospital Affiliated to Shanghai University of Traditional Chinese Medicine (SGKJ-201912).

\section{References}

[1] S. Crotty, "Follicular helper CD4 T cells $\left(\mathrm{T}_{\mathrm{FH}}\right), "$ Annual Review of Immunology, vol. 29, no. 1, pp. 621-663, 2011.

[2] P. Wang, Y. Wang, L. Xie et al., "The transcription factor T-Bet is required for optimal type I follicular helper T cell maintenance during acute viral infection," Frontiers in Immunology, vol. 10, p. 606, 2019.

[3] R. J. Johnston, A. C. Poholek, D. DiToro et al., "Bcl6 and Blimp-1 are reciprocal and antagonistic regulators of $\mathrm{T}$ follicular helper cell differentiation," Science, vol. 325, no. 5943, pp. 1006-1010, 2009.

[4] M. A. Kroenke, D. Eto, M. Locci et al., "Bcl6 and Maf cooperate to instruct human follicular helper CD4 T cell differentiation," The Journal of Immunology, vol. 188, no. 8, pp. 3734-3744, 2012.

[5] X. Liu, X. Chen, B. Zhong et al., "Transcription factor achaete-scute homologue 2 initiates follicular T-helper-cell development," Nature, vol. 507, no. 7493, pp. 513-518, 2014.

[6] F. Andris, S. Denanglaire, M. Anciaux, M. Hercor, H. Hussein, and O. Leo, "The transcription factor c-Maf promotes the differentiation of follicular helper T cells," Frontiers in Immunology, vol. 8, p. 606, 2017.

[7] L. Xu, Y. Cao, Z. Xie et al., "The transcription factor TCF-1 initiates the differentiation of $\mathrm{T}_{\mathrm{FH}}$ cells during acute viral infection," Nature Immunology, vol. 16, no. 9, pp. 991-999, 2015.

[8] R. I. Nurieva, Y. Chung, G. J. Martinez et al., "Bcl6 mediates the development of $\mathrm{T}$ follicular helper cells," Science, vol. 325, no. 5943, pp. 1001-1005, 2009.

[9] D. Yu, S. Rao, L. M. Tsai et al., "The transcriptional repressor Bcl-6 directs T follicular helper cell lineage commitment," Immunity, vol. 31, no. 3, pp. 457-468, 2009.

[10] D. Baumjohann, T. Okada, and K. M. Ansel, "Cutting edge: distinct waves of BCL6 expression during $\mathrm{T}$ follicular helper cell development," The Journal of Immunology, vol. 187, no. 5, pp. 2089-2092, 2011.

[11] Y. S. Choi, R. Kageyama, D. Eto et al., "ICOS receptor instructs $\mathrm{T}$ follicular helper cell versus effector cell differentiation via induction of the transcriptional repressor Bcl6," Immunity, vol. 34, no. 6, pp. 932-946, 2011.

[12] L. Dai, L. He, Z. Wang et al., "Altered circulating T follicular helper cells in patients with chronic immune thrombocytopenia," Experimental and Therapeutic Medicine, vol. 16, no. 3, pp. 2471-2477, 2018.

[13] Z. Wang, M. Zhao, J. Yin et al., "E4BP4-mediated inhibition of $\mathrm{T}$ follicular helper cell differentiation is compromised in autoimmune diseases," Journal of Clinical Investigation, vol. 130, no. article JCI129018, 2020.

[14] Y. Chen, W. Lin, H. Yang et al., "Aberrant expansion and function of follicular helper T cell subsets in IgG4-related disease," Arthritis \& Rheumatology, vol. 70, no. 11, pp. 1853-1865, 2018.

[15] G. Cao, S. Chi, X. Wang, J. Sun, and Y. Zhang, "CD4+CXCR5 $+\mathrm{PD}-1+\mathrm{T}$ follicular helper cells play a pivotal role in the development of rheumatoid arthritis," Medical Science Monitor, vol. 25, pp. 3032-3040, 2019.

[16] Y. Cao, Q. Yang, H. Deng et al., "Transcriptional factor ATF3 protects against colitis by regulating follicular helper $\mathrm{T}$ cells in Peyer's patches," Proceedings of the National Academy of Sciences, vol. 116, no. 13, pp. 6286-6291, 2019. 
[17] T. Maehara, H. Mattoo, V. S. Mahajan et al., "The expansion in lymphoid organs of IL-4 BATF T follicular helper cells is linked to IgG4 class switching in vivo," Life Science Alliance, vol. 1 , no. 1, 2017.

[18] V. Krishnamoorthy, S. Kannanganat, M. Maienschein-Cline et al., "The IRF4 gene regulatory module functions as a readwrite integrator to dynamically coordinate T helper cell fate," Immunity, vol. 47, no. 3, pp. 481-497.e7, 2017.

[19] R. Zhang, C. F. Qi, Y. Hu et al., “T follicular helper cells restricted by IRF8 contribute to $\mathrm{T}$ cell-mediated inflammation," Journal of Autoimmunity, vol. 96, pp. 113-122, 2019.

[20] X. Duan, P. Sun, Y. Lan et al., " 1 IFN- $\alpha$ modulates memory Tfh cells and memory B cells in mice, following recombinant FMDV adenoviral challenge," Frontiers in Immunology, vol. 11, p. 701, 2020.

[21] S. B. Chodisetti, A. J. Fike, P. P. Domeier et al., "Serine phosphorylation of the STAT1 transactivation domain promotes autoreactive B cell and systemic autoimmunity development," The Journal of Immunology, vol. 204, no. 10, pp. 2641-2650, 2020.

[22] X. Ma, S. Nakayamada, S. Kubo et al., "Expansion of T follicular helper-T helper 1 like cells through epigenetic regulation by signal transducer and activator of transcription factors," Annals of the Rheumatic Diseases, vol. 77, no. 9, pp. 13541361, 2018.

[23] J. Deng, C. Fan, X. Gao et al., "Signal transducer and activator of transcription 3 hyperactivation associates with follicular helper T cell differentiation and disease activity in rheumatoid arthritis," Frontiers in Immunology, vol. 9, p. 1226, 2018.

[24] J. S. Weinstein, B. J. Laidlaw, Y. Lu et al., "STAT4 and T-bet control follicular helper T cell development in viral infections," Journal of Experimental Medicine, vol. 215, no. 1, pp. 337-355, 2018.

[25] R. J. Johnston, Y. S. Choi, J. A. Diamond, J. A. Yang, and S. Crotty, "STAT5 is a potent negative regulator of $\mathrm{T}_{\mathrm{FH}}$ cell differentiation," Journal of Experimental Medicine, vol. 209, no. 2, pp. 243-250, 2012.

[26] R. I. Nurieva, A. Podd, Y. Chen et al., "STAT5 protein negatively regulates $\mathrm{T}$ follicular helper ( $\mathrm{Tfh}$ ) cell generation and function," Journal of Biological Chemistry, vol. 287, no. 14, pp. 11234-11239, 2012.

[27] A. A. Sheikh, L. Cooper, M. Feng et al., "Context-dependent role for T-bet in $\mathrm{T}$ follicular helper differentiation and germinal center function following viral infection," Cell Reports, vol. 28, no. 7, pp. 1758-1772.e4, 2019.

[28] F. Li, Z. Zeng, S. Xing et al., "Ezh2 programs $\mathrm{T}_{\mathrm{FH}}$ differentiation by integrating phosphorylation-dependent activation of Bcl6 and polycomb-dependent repression of p19Arf," Nature Communications, vol. 9, no. 1, p. 5452, 2018.

[29] P. Shao, F. Li, J. Wang, X. Chen, C. Liu, and H. H. Xue, "Cutting edge: Tcf1 instructs $\mathrm{T}$ follicular helper cell differentiation by repressing Blimp1 in response to acute viral infection," The Journal of Immunology, vol. 203, no. 4, pp. 801-806, 2019.

[30] Y. S. Choi, J. A. Gullicksrud, S. Xing et al., "LEF-1 and TCF-1 orchestrate $\mathrm{T}_{\mathrm{FH}}$ differentiation by regulating differentiation circuits upstream of the transcriptional repressor Bcl6," Nature Immunology, vol. 16, no. 9, pp. 980-990, 2015.

[31] W. Xu, X. Zhao, X. Wang et al., "The transcription factor Tox2 drives $\mathrm{T}$ follicular helper cell development via regulating chromatin accessibility," Immunity, vol. 51, no. 5, pp. 826-839.e5, 2019.
[32] K. Otsuka, A. Yamada, M. Saito et al., "Achaete-Scute Homologue 2-Regulated Follicular Helper T Cells Promote Autoimmunity in a Murine Model for Sjögren Syndrome," The American Journal of Pathology, vol. 189, no. 12, pp. 24142427, 2019.

[33] J. Geng, H. Wei, B. Shi et al., "Bach2 negatively regulates T follicular helper cell differentiation and is critical for $\mathrm{CD}^{+}{ }^{+} \mathrm{T}$ cell memory," The Journal of Immunology, vol. 202, no. 10, pp. 2991-2998, 2019.

[34] A. Lahmann, J. Kuhrau, F. Fuhrmann et al., "Bach2 controls T follicular helper cells by direct repression of Bcl-6," The Journal of Immunology, vol. 202, no. 8, pp. 2229-2239, 2019.

[35] M. Xu, F. Wang, H. Chen et al., "Inactivation of FOXO1 induces $\mathrm{T}$ follicular cell polarization and involves angioimmunoblastic T cell lymphoma," Cancer Biology and Medicine, vol. 16, no. 4, pp. 743-755, 2019.

[36] B. Shi, J. Geng, Y. H. Wang et al., "Foxp1 negatively regulates $\mathrm{T}$ follicular helper cell differentiation and germinal center responses by controlling cell migration and CTLA4," The Journal of Immunology, vol. 200, no. 2, pp. 586594, 2018.

[37] I. Serr, R. W. Fürst, V. B. Ott et al., "miRNA92a targets KLF2 and the phosphatase PTEN signaling to promote human T follicular helper precursors in T1D islet autoimmunity," Proceedings of the National Academy of Sciences, vol. 113, no. 43, pp. E6659-E6668, 2016.

[38] J.-Y. Lee, C. N. Skon, J. Y. Lee et al., “The Transcription Factor KLF2 Restrains $\mathrm{CD}^{+}{ }^{\mathrm{T}}$ Follicular Helper Cell Differentiation," Immunity, vol. 42, no. 2, pp. 252-264, 2015.

[39] M. M. Xie, B. H. Koh, K. Hollister et al., "Bcl6 promotes follicular helper T-cell differentiation and PD-1 expression in a Blimp1-independent manner in mice," European Journal of Immunology, vol. 47, no. 7, pp. 1136-1141, 2017.

[40] S. Crotty, "T follicular helper cell differentiation, function, and roles in disease," Immunity, vol. 41, no. 4, pp. 529-542, 2014.

[41] J. A. Yang, N. J. Tubo, M. D. Gearhart, V. J. Bardwell, and M. K. Jenkins, "Cutting edge: Bcl6-interacting corepressor contributes to germinal center $\mathrm{T}$ follicular helper cell formation and B cell helper function," The Journal of Immunology, vol. 194, no. 12, pp. 5604-5608, 2015.

[42] J. P. Nance, S. Bélanger, R. J. Johnston, J. K. Hu, T. Takemori, and S. Crotty, "Bcl6 middle domain repressor function is required for $\mathrm{T}$ follicular helper cell differentiation and utilizes the corepressor MTA3," Proceedings of the National Academy of Sciences, vol. 112, no. 43, pp. 13324-13329, 2015.

[43] Y. S. Choi, D. Eto, J. A. Yang, C. Lao, and S. Crotty, "Cutting edge: STAT1 is required for IL-6-mediated Bcl6 induction for early follicular helper cell differentiation," The Journal of Immunology, vol. 190, no. 7, pp. 3049-3053, 2013.

[44] X. Long, L. Zhang, Y. Zhang et al., "Histone methyltransferase Nsd2 is required for follicular helper T cell differentiation," Journal of Experimental Medicine, vol. 217, no. 1, 2020.

[45] N. Xiao, D. Eto, C. Elly, G. Peng, S. Crotty, and Y. C. Liu, "The E3 ubiquitin ligase Itch is required for the differentiation of follicular helper T cells," Nature Immunology, vol. 15, no. 7, pp. 657-666, 2014.

[46] X. Zhang, R. Dai, W. Li et al., "Abnormalities of follicular helper T-cell number and function in Wiskott-Aldrich syndrome," Blood, vol. 127, no. 25, pp. 3180-3191, 2016.

[47] X. Liu, H. Lu, T. Chen et al., "Genome-wide analysis identifies Bcl6-controlled regulatory networks during $\mathrm{T}$ follicular helper 
cell differentiation," Cell Reports, vol. 14, no. 7, pp. 1735-1747, 2016.

[48] A. Ripamonti, E. Provasi, M. Lorenzo et al., "Repression of miR-31 by BCL6 stabilizes the helper function of human follicular helper T cells," Proceedings of the National Academy of Sciences, vol. 114, no. 48, pp. 12797-12802, 2017.

[49] S. Crotty, R. J. Johnston, and S. P. Schoenberger, "Effectors and memories: Bcl-6 and Blimp-1 in T and B lymphocyte differentiation," Nature Immunology, vol. 11, no. 2, pp. 114-120, 2010.

[50] B. C. Betz, K. L. Jordan-Williams, C. Wang et al., "Batf coordinates multiple aspects of $\mathrm{B}$ and $\mathrm{T}$ cell function required for normal antibody responses," Journal of Experimental Medicine, vol. 207, no. 5, pp. 933-942, 2010.

[51] W. Ise, M. Kohyama, B. U. Schraml et al., "The transcription factor BATF controls the global regulators of class-switch recombination in both B cells and T cells," Nature Immunology, vol. 12, no. 6, pp. 536-543, 2011.

[52] A. Sahoo, A. Alekseev, K. Tanaka et al., "Batf is important for IL-4 expression in T follicular helper cells," Nature Communications, vol. 6, no. 1, article 7997, 2015.

[53] P. Li, R. Spolski, W. Liao et al., "BATF-JUN is critical for IRF4mediated transcription in T cells," Nature, vol. 490, no. 7421, pp. 543-546, 2012.

[54] R. I. Nurieva, Y. Chung, D. Hwang et al., "Generation of T follicular helper cells is mediated by interleukin-21 but independent of T helper 1, 2, or 17 cell lineages," Immunity, vol. 29, no. 1, pp. 138-149, 2008.

[55] M. Huber, A. Brustle, K. Reinhard et al., "IRF4 is essential for IL-21-mediated induction, amplification, and stabilization of the Th17 phenotype," Proceedings of the National Academy of Sciences, vol. 105, no. 52, pp. 20846-20851, 2008.

[56] C. S. Ma, D. T. Avery, A. Chan et al., "Functional STAT3 deficiency compromises the generation of human $\mathrm{T}$ follicular helper cells," Blood, vol. 119, no. 17, pp. 3997-4008, 2012.

[57] N. Schmitt, Y. Liu, S. E. Bentebibel et al., "The cytokine TGF- $\beta$ co-opts signaling via STAT3-STAT4 to promote the differentiation of human $\mathrm{T}_{\mathrm{FH}}$ cells," Nature Immunology, vol. 15, no. 9, pp. 856-865, 2014.

[58] K. A. Read, M. D. Powell, C. E. Baker et al., "Integrated STAT3 and Ikaros zinc finger transcription factor activities regulate Bcl-6 expression in CD4 $4^{+}$Th cells," The Journal of Immunology, vol. 199, no. 7, pp. 2377-2387, 2017.

[59] J. Wei, Y. Yuan, C. Jin et al., "The ubiquitin ligase TRAF6 negatively regulates the JAK-STAT signaling pathway by binding to STAT3 and mediating its ubiquitination," PLoS One, vol. 7, no. 11, article e49567, 2012.

[60] P. W. McDonald, K. A. Read, C. E. Baker et al., "IL-7 signalling represses Bcl-6 and the $\mathrm{T}_{\mathrm{FH}}$ gene program," Nature Communications, vol. 7, no. 1, article 10285, 2016.

[61] X. Lin, X. Wang, F. Xiao et al., "IL-10-producing regulatory B cells restrain the $\mathrm{T}$ follicular helper cell response in primary Sjogren's syndrome," Cellular \& Molecular Immunology, vol. 16, no. 12, pp. 921-931, 2019.

[62] L. He, W. Gu, M. Wang et al., "Extracellular matrix protein 1 promotes follicular helper $\mathrm{T}$ cell differentiation and antibody production," Proceedings of the National Academy of Sciences, vol. 115, no. 34, pp. 8621-8626, 2018.

[63] J. Zhang, Z. He, S. Sen, F. Wang, Q. Zhang, and Z. Sun, “TCF-1 inhibits IL-17 gene expression to restrain Th17 immunity in a stage-specific manner," The Journal of Immunology, vol. 200, no. 10 , pp. 3397-3406, 2018.
[64] J. Hosokawa, K. Suzuki, K. Meguro et al., "I $\kappa$ BNS enhances follicular helper T-cell differentiation and function downstream of ASCl2," Journal of Allergy and Clinical Immunology, vol. 140, no. 1, pp. 288-291.e8, 2017.

[65] H. Qiu, H. Wu, V. Chan, C. S. Lau, and Q. Lu, “Transcriptional and epigenetic regulation of follicular T-helper cells and their role in autoimmunity," Autoimmunity, vol. 50, no. 2, pp. 7181, 2017.

[66] H. Zeng, S. Cohen, C. Guy et al., "mTORC1 and mTORC2 kinase signaling and glucose metabolism drive follicular helper T cell differentiation," Immunity, vol. 45, no. 3, pp. 540-554, 2016.

[67] H. Wang, J. Geng, X. Wen et al., "The transcription factor Foxp1 is a critical negative regulator of the differentiation of follicular helper T cells," Nature Immunology, vol. 15, no. 7, pp. 667-675, 2014. 